\title{
Intervenções educativas desenvolvidas por enfermeiros para pessoas com Diabetes
}

\section{mellitus}

\author{
Educational interventions developed by nurses for people with Diabetes mellitus \\ Intervenciones educativas desarrolladas por enfermeras para personas con Diabetes mellitus
}

Recebido: 20/08/2021 | Revisado: 29/08/2021 | Aceito: 03/09/2021 | Publicado: 05/09/2021

\author{
Ruth Helena Lopes Rodrigues \\ ORCID: https://orcid.org/0000-0002-1826-0746 \\ Universidade Federal do Pará, Brasil \\ E-mail: ruthhelena.2609@gmail.com \\ Mary Elizabeth de Santana \\ ORCID: https://orcid.org/0000-0002-3629-8932 \\ Universidade Federal do Pará, Brasil \\ E-mail: mary.santana@uepa.br
}

\begin{abstract}
Resumo
Objetivo: caracterizar as evidências científicas encontradas na literatura sobre as intervenções educativas desenvolvidas por enfermeiros para pessoas com diabetes mellitus. Metodologia: trata-se de estudo de revisão integrativa que tem por escopo caracterizar as pesquisas já realizadas sobre as intervenções educativas desenvolvidas por enfermeiros para pessoas com diabetes mellitus, para tanto buscou-se utilizar a estratégia PICO, para a construção da questão norteadora: Quais são as evidências disponíveis na literatura sobre as intervenções educativas desenvolvidas por enfermeiros para a pessoa com diabetes mellitus? A busca ocorreu nas bases de dados que são Literatura Latino-Americana e do Caribe em Ciências da Saúde (LILACS); MedLine; PubMed; Scientific Electronic Library Online (SciELO) e Base de Dados de Enfermagem (BDENF). Resultados: dez $(n=10)$ estudos foram incluídos nesta revisão, estes foram compreendidos no período de 2016 a 2020, a maioria das publicações utilizadas neste estudo $(n=6)$ foi publicado em português e teve sua origem no Brasil, o que demonstra certa fragilidade e lacuna na publicação internacional em relação à temática. Quanto aos níveis de evidência verificou-se a predominância de estudos de nível VI. Conclusão: a revisão das produções acerca da temática evidenciou que os estudos desenvolvidos por enfermeiros sobre a educação do paciente diabético como tema de pesquisa, ainda é pouco explorada em níveis de maior evidência científica. Necessitando de aprofundamento nos métodos de pesquisa para as produções desenvolvidas com esta temática.
\end{abstract}

Palavras-chave: Diabetes mellitus; Cuidados de enfermagem; Educação de pacientes como assunto.

\begin{abstract}
Objective: to characterize the scientific evidence found in the literature on educational interventions developed by nurses for people with diabetes mellitus. Methodology: this is an integrative review study that aims to characterize the research already carried out on educational interventions developed by nurses for people with diabetes mellitus. For this, we sought to use the PICO strategy to build the guiding question: Which are the evidence available in the literature on educational interventions developed by nurses for people with diabetes mellitus? The search took place in the databases that are Latin American and Caribbean Literature in Health Sciences (LILACS); MedLine; PubMed; Scientific Electronic Library Online (SciELO) and Nursing Database (BDENF). Results: ten $(\mathrm{n}=10)$ studies were included in this review, these were comprised in the period from 2016 to 2020, most of the publications used in this study $(n=6)$ were published in Portuguese and originated in Brazil, which demonstrates certain weakness and gap in the international publication in relation to the theme. As for the levels of evidence, there was a predominance of level VI studies. Conclusion: the review of productions on the subject showed that the studies developed by nurses on the education of diabetic patients as a research topic, is still little explored in levels of greater scientific evidence. Needing further research methods for productions developed with this theme.
\end{abstract}

Keywords: Diabetes mellitus; Nursing care; Patient education as a subject.

\section{Resumen}

Objetivo: caracterizar la evidencia científica encontrada en la literatura sobre intervenciones educativas desarrolladas por enfermeras para personas con diabetes mellitus. Metodología: se trata de un estudio de revisión integradora que tiene como objetivo caracterizar la investigación ya realizada sobre intervenciones educativas desarrolladas por enfermeras para personas con diabetes mellitus, para lo cual se buscó utilizar la estrategia PICO para construir la pregunta orientadora: ¿Cuáles son las evidencias disponibles en la literatura sobre intervenciones educativas 
desarrolladas por enfermeras para personas con diabetes mellitus? La búsqueda se realizó en las bases de datos de Literatura Latinoamericana y Caribeña en Ciencias de la Salud (LILACS); MedLine; PubMed; Scientific Electronic Library Online (SciELO) y Base de datos de enfermería (BDENF). Resultados: se incluyeron diez $(\mathrm{n}=10)$ estudios en esta revisión, estos fueron comprendidos en el período de 2016 a 2020, la mayoría de las publicaciones utilizadas en este estudio $(n=6)$ fueron publicadas en portugués y tuvieron su origen en Brasil, lo que demuestra cierta debilidad y brecha en la publicación internacional en relación al tema. En cuanto a los niveles de evidencia, predominaron los estudios de nivel VI. Conclusión: la revisión de producciones sobre el tema mostró que los estudios desarrollados por enfermeras sobre la educación del paciente diabético como tema de investigación, aún está poco explorado en niveles de mayor evidencia científica. Se necesitan más métodos de investigación para las producciones desarrolladas con este tema.

Palabras clave: Diabetes mellitus; Atención de enfermería; La educación del paciente como asunto.

\section{Introdução}

Diabetes mellitus (DM) é uma condição clínica definida como um grupo de doenças metabólicas caracterizadas por hiperglicemia resultante de defeitos na secreção ou na ação da insulina. A hiperglicemia crônica está associada a complicações a longo prazo, incluindo a retinopatia diabética com potencial perda da visão, neuropatias, nefropatias, feridas diabéticas e amputação de membros inferiores, além de sintomas cardiovasculares e disfunções sexuais (American Diabetes Association, 2020).

Estima-se que o diabetes tem prevalência mundial entre $8,8 \%$ e $14,2 \%$, enquanto no Brasil se tem como realidade epidemiológica o aumento na prevalência desta doença, que passou de 5,5\% para 7,4\% (American Diabetes Association, 2020; Brasil, 2013). As pessoas com Diabetes mellitus necessitam de cuidados de saúde especializados por se tratar de doentes crônicos, com propensão a desenvolvimento de complicações da doença. O estudo de Silva, et al. (2020) identificou o perfil epidemiológico de pessoas com diabetes, cuja predominância foi de mulheres, na faixa etária entre a 60 a 69 anos, com ensino fundamental incompleto, fatores estes primordiais para o desenvolvimento de complicações do diabetes, por conta das dificuldades que este público apresenta em realizar e compreender as ações de autocuidado em relação ao controle do diabetes.

Segundo a Sociedade Brasileira de Diabetes (2019), especificamente no Brasil a despesa anual com o tratamento ambulatorial por paciente com DM pelo Sistema Único de Saúde (SUS) varia em torno de R\$10.814,00, sendo que R\$ 6.848,00 $(63,3 \%)$ são referentes aos custos diretos, calcula-se que os gastos com saúde de indivíduos com diabetes sejam 2 a 3 vezes maiores do que daqueles sem diabetes. Ressaltando que o custo avaliado em 2015 foi de R 112 bilhões, com projeção de R\$ 148 bilhões para 2040, sendo que $91 \%$ desse valor são referentes as internações hospitalares.

A descompensação do quadro clínico é a principal causa de internação hospitalar por diabetes, e pode ter origem na falta de adaptação ao tratamento e as dificuldades no autogerenciamento do cuidado em saúde enfrentadas por estas pessoas (Frota, et al., 2020). No que diz respeito ao autogerenciamento do cuidado em saúde, os enfermeiros são os profissionais de saúde que utilizam-se de práticas e de tecnologias educativas para a promoção de saúde e prevenção de doenças, para o cuidado de um indivíduo em todas as fases da sua vida, e quanto maior as necessidades de cuidado deste indivíduo maior são as necessidades de intervenções educativas, em se tratando de necessidades de cuidado, os pacientes com doenças crônicas necessitam de um bom autogerenciamento dos fatores de risco que desencadearão em internações hospitalares por complicações do diabetes. Para resolver esses problemas e viver bem com diabetes, requer um bom nível de cuidados de saúde e de relação entre os profissionais de saúde e o indivíduo (Wilson, 2021).

Para o bom gerenciamento do cuidado destas pessoas, temos as práticas educativas, cujas necessitam que estejam voltadas para o autocuidado e autogerenciamento da saúde de pessoas com Diabetes mellitus, sendo primordial que estas ações abordem a mudança de comportamento e empoderamento sobre o controle do diabetes. O autocuidado no diabetes envolve o desenvolvimento do conhecimento e da consciência, aprendendo a conviver com a complexidade da doença em um contexto 
social. As intervenções destinadas a encorajar a motivação do paciente e a confiança em seus comportamentos de saúde são esporádicas, e menos ainda foram direcionadas especificamente para o autocuidado com diabetes (Swanson \& Maltinsky, 2019).

Nesse sentido é de fundamental importância a atuação do profissional de Enfermagem para o empoderamento dessas pessoas no diz respeito ao desenvolvimento de práticas de promoção e prevenção de complicações para a melhoria da qualidade de vida, e principalmente para a adaptação, reabilitação e enfrentamento destes pacientes diante da doença e das novas condições de vida mediante ao desenvolvimento de complicações. Diante disso questiona-se: Quais são as evidências científicas encontradas na literatura sobre as intervenções educativas desenvolvidas por enfermeiros para pessoas com Diabetes mellitus?

Com isso, o objetivo desse estudo foi caracterizar as evidências científicas encontradas na literatura sobre as intervenções educativas desenvolvidas por enfermeiros para pessoas com Diabetes mellitus, assim como caracterizar as tecnologias educativas desenvolvidas e/ou utilizadas por enfermeiros para mediar o processo de autogerenciamento do cuidado de pessoas com Diabetes mellitus.

\section{Metodologia}

Trata-se de estudo de revisão integrativa que tem por escopo resumir as pesquisas já realizadas e estabelecer a partir de uma avaliação crítica com a meta de sintetizar e analisar os dados para desenvolver uma explicação mais ampla de um fenômeno específico, com a meta de síntese e análise dos achados nos estudos (Mendes; Silveira \& Galvão, 2008).

Adotou-se as seis etapas para construção desta revisão: seleção da pergunta de pesquisa; definição dos critérios de inclusão e exclusão de estudos e seleção da amostra; estudos selecionados; considerando as características; análise crítica dos achados; interpretação dos resultados e reportar, de forma clara, a evidência identificada (Whittemore \& Knafl, 2005).

Para a elaboração da pergunta norteadora a estratégia PICO será usada, sendo P de população, paciente ou problema (pacientes com Diabetes mellitus), I de intervenções (ações educativas realizadas por enfermeiros), C de comparação (ausência das intervenções educativas) e $\mathrm{O}$ de desfechos (os resultados dessas ações para o controle do diabetes).

Dessa maneira, esse estudo buscará responder a seguinte questão norteadora: Quais são as evidências disponíveis na literatura sobre as intervenções educativas desenvolvidas por enfermeiros para a pessoa com Diabetes mellitus?

Foram incluídos os estudos publicados em inglês, espanhol e português, sobre temática as intervenções educativas desenvolvidas por enfermeiros para a pessoa com Diabetes mellitus do tipo revisões sistemáticas, estudos descritivos, exploratórios, retrospectivos e estudos de coorte. E excluídos os estudos que estiverem fora do período temporal e temática estabelecidos na busca.

Nesta etapa foram estabelecidos os Descritores em saúde (DeCS, em se tratando da busca de produções nacionais e MeSH para a busca de produções internacionais) para mediar a busca da literatura foram utilizados os operadores booleanos AND e OR, para tanto definiu-se a utilização dos seguintes descritores: "Diabetes mellitus" (em inglês: "Diabetes mellitus"; em espanhol: "Diabetes mellitus"); "Cuidados de enfermagem" (em inglês: "Nursing care", em espanhol: "Atención de enfermería"); "Educação de pacientes como assunto" (em inglês: "Patient education as a subject ", "em espanhol: "Educación del paciente como asunto"). O período temporal de artigos publicados foi de 2016 a 2020. As buscas das evidências científicas ocorreram nas seguintes bases de dados: Literatura Latino-Americana e do Caribe em Ciências da Saúde (LILACS); Medical Literature Analysis and Retrieval Sisten On-line (MedLine)/ PubMed (via National Library of Medicine); Scientific Electronic Library Online (SciELO) e Base de Dados de Enfermagem (BDENF). As estratégias de busca nas bases de dados se deram segundo a combinação dos descritores e podem ser vistas no Quadro 1. 
Quadro 1. Estratégia de busca nas bases de dados. Belém, Brasil, 2021.

\begin{tabular}{|c|c|}
\hline MEDLINE/PUBMED & $\begin{array}{l}\text { ("Diabetes mellitus" [MeSH Terms] ("Diabetes mellitus" OR "Nursing care" OR "Patient education as a } \\
\text { subject", "Diabetes mellitus" OR "Atencíon de enfermería" OR "Educación del paciente como asunto", } \\
\text { "Diabetes mellitus" AND "Cuidados de enfermagem" AND "Educação do paciente como assunto", "Diabetes } \\
\text { mellitus" OR "Atencíon de enfermería" AND "Patient education as a subject" AND (English [lang] OR } \\
\text { Portuguese [lang] OR Spanish [lang]) }\end{array}$ \\
\hline LILACS/BDENF & 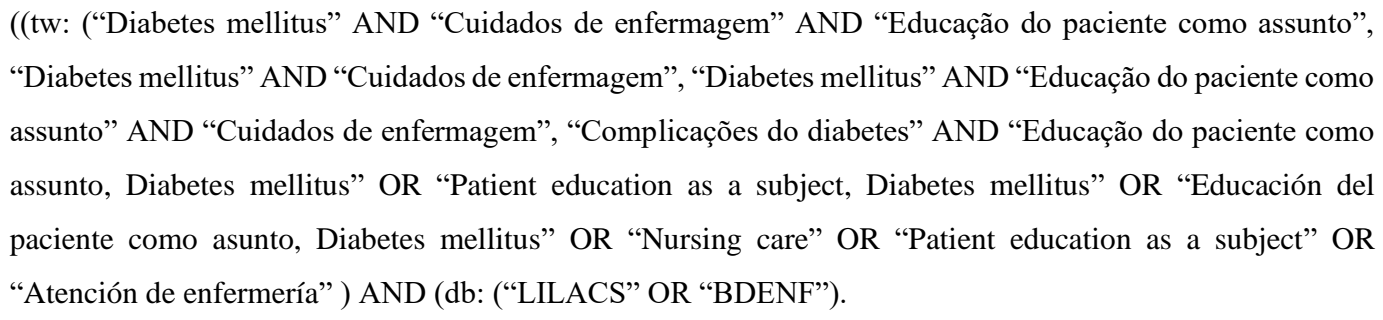 \\
\hline SCIELO & $\begin{array}{l}\text { "Diabetes mellitus" AND "Cuidados de enfermagem" AND "Educação do paciente como assunto", "Diabetes } \\
\text { mellitus" AND "Cuidados de enfermagem", "Diabetes mellitus" AND "Educação do paciente como assunto" } \\
\text { AND "Cuidados de enfermagem", "Complicações do diabetes" AND "Educação do paciente como assunto". }\end{array}$ \\
\hline
\end{tabular}

Fonte: Autores.

Após a coleta nas bases de dados, os dados coletados foram organizados e filtrados em um software online (Rayyan).

Mediante a seleção e filtragem dos artigos encontrados na busca realizada na etapa anterior do estudo, os dados do processo são apresentados por intermédio de fluxograma explicativo Hopia, et al. (2016), segundo as recomendações do protocolo PRISMA, o qual pode ser visualizado na Figura 1. A análise dos dados foi realizada em sete níveis: Nível I (Estudos de revisão sistemática ou metanálise de ensaios clínicos randomizados controlados); Nível II (Ensaio clínico randomizado controlado bem delineado); Nível III (Estudos de ensaios clínicos bem delineados sem randomização); Nível IV (Estudos de corte e de caso-controle bem delineados (não experimentais); Nível V (Estudos de revisão sistemática de estudos descritivos e qualitativos); Nível VI (Evidências derivadas de um único estudo descritivo ou qualitativo); Nível VII (Evidências oriundas de opinião de autoridades ou comitês) (Melnyk, et al., 2010). 
Figura 1. Fluxograma dos cruzamentos e resultados de busca, segundo as recomendações do protocolo PRISMA. Belém, Brasil, 2021.

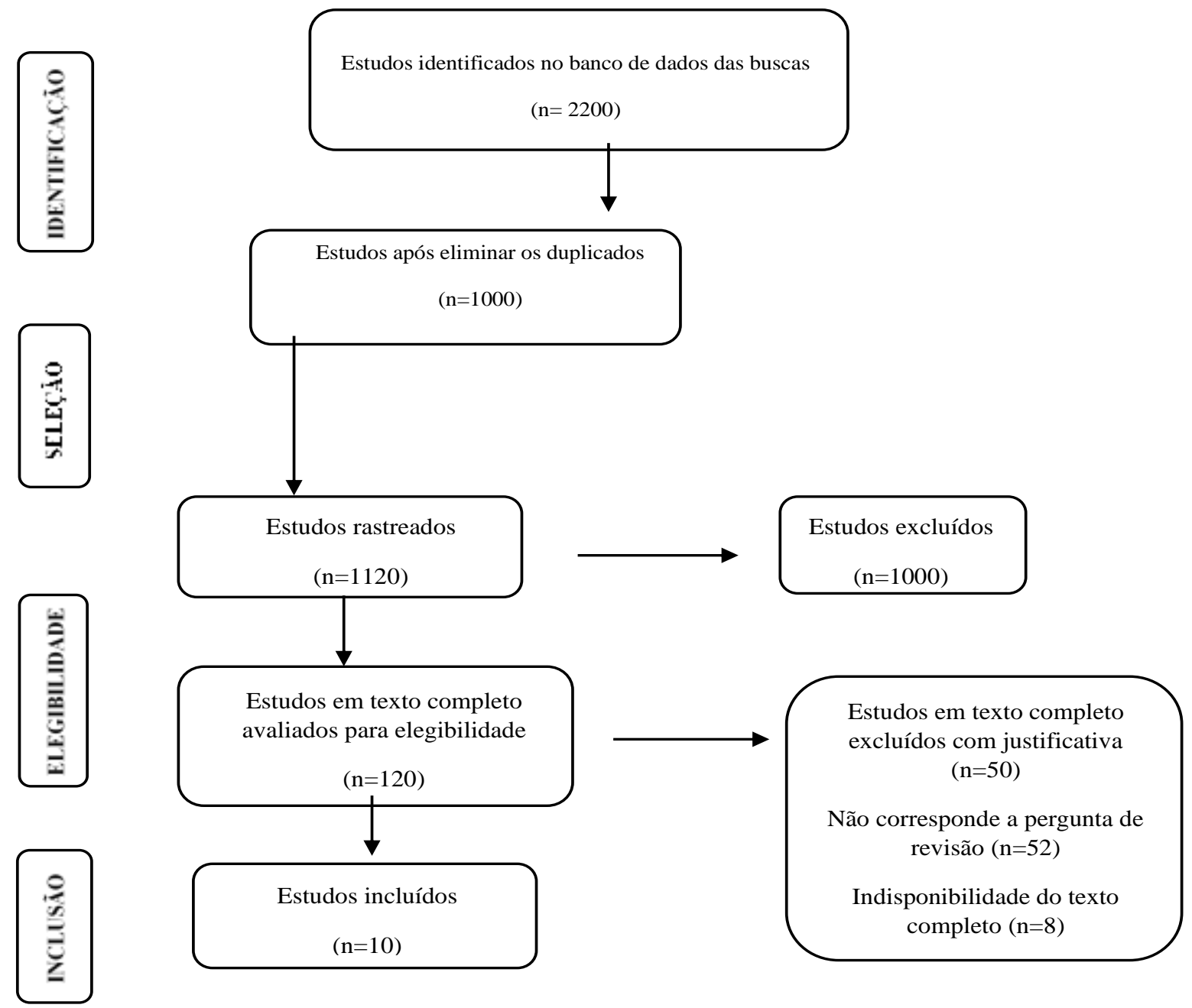

Fonte: Autores.

\section{Resultados e Discussão}

Dez estudos foram selecionados nesta revisão, estes foram compreendidos no período de 2016 a 2020, dentre os critérios de inclusão presentes neste estudo as línguas inglesa e portuguesa foram selecionadas, no entanto nenhum artigo da língua espanhola foi incluído, entretanto um artigo de origem espanhola foi encontrado na busca, porém publicado na língua inglesa. A maioria dos estudos utilizados $(n=6)$ foram publicados em português e tiveram sua origem no Brasil, o que demonstra certa fragilidade e lacuna na publicação internacional em relação à temática.

Quanto aos níveis de evidência verificou-se a predominância de estudos de nível VI com enfoque em estudos descritivos não experimentais diante da temática. No entanto, apesar disto pôde-se identificar um estudo nacional com nível de evidência II. No Quadro 2 estão apresentados os dados dos estudos analisados segundo as variáveis: autores, país, ano de publicação, objetivos, nível de evidência e base de dados. Em seguida no Quadro 3 podem ser encontradas as principais ações de enfermagem encontradas na literatura a respeito. 
Quadro 2. Estudos encontrados conforme autoria, país, ano de publicação, objetivos, nível de evidência e base de dados.

\begin{tabular}{|c|c|c|c|c|}
\hline AUTORIA & $\begin{array}{l}\text { PAÍS DE ORIGEM } \\
\text { E ANO DE } \\
\text { PUBLICAÇÃO }\end{array}$ & OBJETIVOS & $\begin{array}{l}\text { NÍVEL DE } \\
\text { EVIDÊNCIA }\end{array}$ & BASE DE DADOS \\
\hline Torres, et al. & $\begin{array}{c}\text { Brasil } \\
2016\end{array}$ & $\begin{array}{l}\text { Avaliar a educação em grupo para o controle metabólico } \\
\text { dos usuários com diabetes mellitus } 2 \text { na Atenção Primária } \\
\text { à Saúde. }\end{array}$ & Nível II & BDENF \\
\hline $\begin{array}{l}\text { Carvalho, et } \\
\text { al. }\end{array}$ & $\begin{array}{l}\text { rasil } \\
2018\end{array}$ & $\begin{array}{l}\text { Relatar a experiência da utilização de mapas de } \\
\text { conversação em diabetes como estratégia educativa ao } \\
\text { idoso diabético. }\end{array}$ & Nível VI & SCIELO \\
\hline Silva, et al. & $\begin{array}{c}\text { Brasil } \\
2018\end{array}$ & $\begin{array}{l}\text { Tem como objetivo a construção e validação de um } \\
\text { simulador de baixo custo para uso na capacitação de } \\
\text { pacientes com diabetes mellitus e de seus cuidadores para } \\
\text { a aplicação de insulina. }\end{array}$ & Nível VI & LILACS \\
\hline Galdino, et al. & $\begin{array}{c}\text { Brasil } \\
2019\end{array}$ & $\begin{array}{l}\text { Descrever a validação de cartilha sobre o autocuidado } \\
\text { com pés de pessoas com diabetes. }\end{array}$ & Nível VI & SCIELO \\
\hline Baptista, et al. & $\begin{array}{c}\text { Brasil } \\
2019\end{array}$ & $\begin{array}{l}\text { Avaliar as contribuições de um programa educativo para } \\
\text { a automonitorização da glicemia capilar. }\end{array}$ & Nível III & SCIELO \\
\hline $\begin{array}{l}\text { Marques, et } \\
\quad \text { al. }\end{array}$ & $\begin{array}{c}\text { Brasil } \\
2019\end{array}$ & $\begin{array}{l}\text { Avaliar a eficácia de uma intervenção educativa de } \\
\text { enfermagem para o autocuidado em idosos com DM. }\end{array}$ & Nível III & SCIELO \\
\hline Vest, et al. & $\begin{array}{l}\text { EUA } \\
2017\end{array}$ & $\begin{array}{l}\text { Relatar a experiência de implementação de um programa } \\
\text { de telemonitoramento de pessoas com diabetes como } \\
\text { rotina em ambientes de cuidados primários. }\end{array}$ & Nível VI & PUBMED/MEDLINE \\
\hline $\begin{array}{l}\text { De La Fuente } \\
\text { Coria, et al.. }\end{array}$ & $\begin{array}{c}\text { Espanha } \\
2020\end{array}$ & $\begin{array}{l}\text { Avaliar a eficácia de um programa de educação } \\
\text { estruturado e individualizado para pessoas com diabetes, } \\
\text { que contou com reforços educacionais e apoio familiar } \\
\text { para alcançar o controle metabólico e metas terapêuticas } \\
\text { de longo prazo. }\end{array}$ & Nível II & PUBMED/MEDLINE \\
\hline Kho, et al. & $\begin{array}{c}\text { Singapura } \\
2018\end{array}$ & $\begin{array}{l}\text { Desenvolver e testar a usabilidade e viabilidade de um } \\
\text { aplicativo móvel educativo para pacientes com diabetes } \\
\text { mellitus. }\end{array}$ & Nível VI & PUBMED/MEDLINE \\
\hline Smith, et al. & $\begin{array}{l}\text { EUA } \\
2018\end{array}$ & $\begin{array}{l}\text { Examinar as barreiras e facilitadores da integração da } \\
\text { educação em habilidades de sobrevivência em diabetes } \\
\text { (DSSE) via iPad ao fluxo de trabalho da unidade de } \\
\text { internação de enfermagem. }\end{array}$ & Nível VI & PUBMED/MEDLINE \\
\hline
\end{tabular}

Fonte: Autores. Belém, Brasil (2021). 
Quadro 3. Principais ações de enfermagem dos estudos selecionados.

\begin{tabular}{|c|c|}
\hline AUTORIA & PRINCIPAIS AÇÕES/ DESFECHO DAS INTERVENÇÕES/ TECNOLOGIAS IDENTIFICADAS. \\
\hline Torres, et al. & $\begin{array}{l}\text { O acompanhamento dos achados da educação em grupo mostraram aumento nos escores do teste de conhecimentos, } \\
\text { atitudes e do autogerenciamento dos cuidados, ressaltando a redução nos níveis de HbA1c com significância estatística. }\end{array}$ \\
\hline $\begin{array}{l}\text { Carvalho, et } \\
\text { al. }\end{array}$ & $\begin{array}{l}\text { A utilização do mapa de conversação em diabetes permitiu observar a visão que os usuários têm sobre a própria doença, } \\
\text { construindo saberes e práticas para o próprio cotidiano. Foram abordados temas presentes na vida do diabético, como a } \\
\text { fisiopatologia da doença, o tratamento, as complicações, a alimentação, os exercícios, os cuidados com os pés, os } \\
\text { problemas psicológicos, entre outros. }\end{array}$ \\
\hline Silva, $\epsilon$ & $\begin{array}{l}\text { O simulador apresentado no estudo é uma ferramenta que pode facilitar o processo de capacitação para o autocuidado. } \\
\text { Apresenta baixo custo e maior fidelidade anatômico funcional, que os modelos disponíveis no mercado. }\end{array}$ \\
\hline Galdino, et al. & $\begin{array}{l}\text { A participação de pessoas com DM de diferentes níveis de instrução possibilitou avaliar que a tecnologia desenvolvida } \\
\text { está adequada em relação à clareza e compreensão da linguagem e das ilustrações, sendo o conteúdo considerado relevante } \\
\text { para transmissão de conhecimentos e sensibilização à promoção de mudança de comportamentos. }\end{array}$ \\
\hline Baptista, et al. & $\begin{array}{l}\text { Após a participação das pessoas com DM no programa educativo, observaram-se melhora significativa nos valores da } \\
\text { glicemia pós-prandial; na interpretação da glicemia capilar como resultado da interação da alimentação e medicamentos; } \\
\text { no reconhecimento do sintoma "fraqueza" para a hiperglicemia; e na administração dos medicamentos de forma correta } \\
\text { para a prevenção da hiperglicemia. }\end{array}$ \\
\hline Marques, et al. & $\begin{array}{l}\text { A realização das intervenções educativas em uma abordagem grupal evidenciou melhoria nos aspectos da alimentação } \\
\text { saudável e do cuidado com os pés, antes não promovidas conforme verificado no grupo-controle. }\end{array}$ \\
\hline Vest, & $\begin{array}{l}\text { O telemonitoramento pode oferecer benefícios para o monitoramento preventivo de pacientes em risco no ambiente de } \\
\text { atenção primária. No entanto, sua implementação no ambiente das práticas de atenção primária precisa ser planejada } \\
\text { cuidadosamente a fim de maximizar os benefícios da intervenção. }\end{array}$ \\
\hline $\begin{array}{l}\text { De La Fuente } \\
\text { Coria, et al. }\end{array}$ & $\begin{array}{l}\text { A intervenção educativa fornecida por enfermeiras de cuidados primários ao longo de seis meses, com sessões de reforço } \\
\text { após } 12 \text { e } 24 \text { meses, juntamente com o apoio familiar pode alcançar reduções na hemoglobina glicada, glicemia de jejum, } \\
\text { colesterol total, colesterol de lipoproteína de baixa densidade e pressão arterial sistólica no médio e longo prazo. Podendo } \\
\text { ser interpretada como uma forma de retardar a progressão da doença. }\end{array}$ \\
\hline Kho, et al. & $\begin{array}{l}\text { Os resultados deste estudo confirmaram que a tecnologia desenvolvida era utilizável e viável para educação em diabetes. } \\
\text { Ele permitiu aos usuários revisitar ou acessar informações sobre diabetes em seu próprio ritmo, nível de conforto e tempo. }\end{array}$ \\
\hline Smith, et al. & $\begin{array}{l}\text { Existem desafios e oportunidades para implementar uma tecnologia educativa para pessoas com diabetes por meio de um } \\
\text { tablet, para que haja sucesso neste tipo de estratégia é necessário considerar, planejar e validar o conteúdo educacional, } \\
\text { usabilidade da plataforma, viabilidade do tablet associando-os com o fluxo de trabalho de enfermagem nas unidades de } \\
\text { internação. }\end{array}$ \\
\hline
\end{tabular}

Fonte: Autores. Belém, Brasil (2021).

Os principais resultados dos estudos selecionados nesta revisão foram organizados segundo similaridade semântica e derem origem a duas categorias temáticas, apresentadas a seguir: 


\section{Experiências educativas exitosas voltadas para o autogerenciamento do cuidado de pessoas com diabetes}

Torres, et al. (2016); Carvalho, et al. (2018); Baptista, et al. (2019); Marques, et al. (2019) utilizaram como estratégia ações educativas em grupo para abordar temas presentes na vida do diabético, como a fisiopatologia da doença, o tratamento, as complicações, a alimentação, os exercícios, os cuidados com os pés, os problemas psicológicos, entre outros. As práticas educativas realizadas com idosos e familiares, por meio dos mapas de conversação, favoreceram a integração da equipe multidisciplinar com os participantes, proporcionando um ambiente dinâmico e empático para a troca de experiências, elucidação de dúvidas e mudanças comportamentais no cotidiano para o controle do diabetes (Carvalho, et al., 2018).

As intervenções educativas realizadas nos estudos de Torres, et al. (2016); Carvalho, et al. (2018); Baptista, et al. (2019) foram fundamentais para a redução dos índices de HbA1c dos participantes após a adesão e participação frequente dos usuários nos grupos de apoio. Outro aspecto interessante identificado acerca do êxito das ações educativas por meio de grupos foi no estudo de Baptista, et al. (2019) onde o número de participantes que compartilhavam lancetador (caneta) diminuiu de 8 $(38,1 \%)$ para $3(12,5 \%)$.

Pelo fato do acompanhamento regular destes clientes e da melhor compreensão deles em relação a doença, por meio do compartilhamento de experiências, da criação de novos laços e relações de suporte, os idosos participantes nestas pesquisas tiveram impactos positivos em outras áreas além do diabetes, como por exemplo, houve redução no índice de massa corporal destes idosos, dos níveis de adesão da terapia medicamentosa, dos padrões de pressão arterial, bem como houve uma maior predisposição para o desenvolvimento do autocuidado. Estratégias de ensino-aprendizagem pautadas no processo de empoderamento são essenciais para facilitar e dar suporte aos usuários na adesão as práticas de autocuidado (Wermeling, et al., 2014).

O estudo de De La Fuente Coria, et al. (2020) utiliza a abordagem educativa de maneira individualizada, a intervenção consistiu em 6 sessões presenciais com duração de 30 minutos. A intervenção foi realizada por uma enfermeira com mais de 10 anos de experiência em educação em Diabetes mellitus. As sessões educacionais foram ministradas em um período de 6 meses, com reforços educacionais após 12 e 24 meses.

O participante deveria comparecer às sessões acompanhado de um familiar cuidador, para que ambos obtivessem conhecimento básico sobre o controle do diabetes por meio do desenvolvimento de práticas de alimentação saudável, atividade física, automonitoramento de glicose no sangue, adesão aos medicamentos e resolução de problemas e enfrentamento eficaz. Este estudo constatou que a intervenção educativa teve efeitos favoráveis a médio e longo prazo sobre a glicemia de jejum, hemoglobina glicada, colesterol total, colesterol de lipoproteína de baixa densidade e pressão arterial sistólica. Da mesma forma, o objetivo terapêutico que mostrou melhorias significativas em longo prazo com a intervenção educacional foi a hemoglobina glicada (De La Fuente Coria, et al., 2020).

Independentemente se acompanhado em grupo, ou individualmente os participantes dos estudos de Torres, et al. (2016); Carvalho, et al. (2018); Baptista, et al. (2019); Marques, et al. (2019); De La Fuente Coria, et al. (2020) apontaram que o desenvolvimento e adequação do conhecimento de pessoas com Diabetes mellitus mediado por processos educativos de Enfermeiros foi relevante para a melhoria dos índices de saúde destas pessoas, auxiliando na minimização do risco de desenvolvimento de complicações do diabetes e, por conseguinte do risco de internações hospitalares do público que recebeu estas intervenções.

Atualmente, as intervenções mais eficazes para o controle do diabetes estão centradas na mudança de hábitos alimentares, prática regular de atividade física, no controle medicamentoso da glicemia, pressão arterial e lipídios no sangue, e da realização de exames periódicos para detectar precocemente lesão ocular, renal ou nos pés. Todas essas medidas podem prevenir ou retardar as complicações do diabetes, segundo a inclusão dessas práticas em programas de educação em diabetes, 
com o objetivo de melhorar o conhecimento e o comportamento das pessoas em relação ao autogerenciamento do diabetes (Powers, et al., 2015).

\section{Recursos tecnológicos utilizados para mediar o processo educativo de pessoas com diabetes}

No que diz respeito ao desenvolvimento e utilização de recursos tecnológicos para mediar o processo educativo de pessoas com diabetes os estudos de Vest, et al. (2017); Kho, et al. (2019); Smith, et al. (2019); Silva, et al. (2018); Galdino, et al. (2019) abordam diferentes perspectivas e abordagens do uso de tecnologias para a educação em saúde de pessoas com diabetes.

O recurso tecnológico utilizado por Vest, et al. (2017) para realizar ações educativas de pessoas com diabetes são os telefones, e por meio da prática do Telemonitoramento, as enfermeiras educaram os pacientes sobre como verificarem diariamente o peso, a pressão arterial e a glicose, e posteriormente a isso enviar esses resultados para a agência de telemonitoramento. Se qualquer uma dessas medidas estivesse fora dos parâmetros predeterminados estabelecidos pelos médicos dos pacientes, o primeiro curso de ação por todos os enfermeiros era entrar em contato com o paciente e com o familiar cuidador para avaliar a situação. Todas as agências forneceram materiais educacionais impressos aos pacientes como forma de fornecer previamente o conhecimento acerca da necessidade e de como realizar as ações de autocuidado e o monitoramento dos parâmetros de glicose, massa corporal e de pressão arterial. Além disso, as enfermeiras forneciam educação verbal individualizada para as necessidades de cada paciente ao longo de cada encontro, isso favoreceu a evolução dos pacientes em relação ao conhecimento sobre a doença e sobre as ações de autocuidado pois, no início do projeto a abordagem educativa mais intensa, e ao longo do projeto elas foram tomando um caráter de 'atualizações'.

Kho, et al. (2019), utilizou como recurso tecnológico um software em formato de aplicativo para a educação em saúde de pessoas com diabetes. O aplicativo "Diabetes" foi desenvolvido com base na metodologia de Ciclo de Vida de Desenvolvimento de Sistemas Completos (CVDSC), cujo é amplamente utilizado em sistemas de informação e desenvolvimento de software, e forneceram modelos que poderiam ser usados para gerenciar o desenvolvimento de aplicativos de saúde. Nesse sentido, o intuito da tecnologia desenvolvida era motivar e ajudar os pacientes a alcançarem os resultados desejados para o controle glicêmico e quanto ao conhecimento sobre o diabetes e suas complicações. Aplicação de "Diabetes" demonstrou usabilidade e viabilidade em seis domínios: utilidade do sistema, facilidade de uso percebida, atitude em relação ao uso, intenção comportamental de usar, e uso real.

Os participantes concordaram que o aplicativo para diabetes era fácil de usar e trouxeram consciência para a importância do autogerenciamento do diabetes. Foi informativo, melhorou o conhecimento sobre diabetes, desencadeou motivação e comportamentos positivos (como automonitoramento dos níveis de glicose no sangue) e afirmou a crença dos participantes no autocontrole do diabetes. A implementação do aplicativo de "Diabetes" reduziu a duração do aconselhamento sobre diabetes e teve o potencial de reduzir o tempo das consultas de enfermagem pela metade (Kho, et al., 2018).

Smith, et al. (2019) aborda a utilização de tablets no processo educativo pelo diabetes, por intermédio do programa "Diabetes to Go", cujo fornece uma abordagem de aprendizagem adaptativa e foi testado anteriormente para demonstrar eficácia na melhoria da adesão à medicação em pacientes com diabetes. O programa é fornecido em inglês em um dispositivo habilitado para web. Os pacientes primeiro se conectam à plataforma e preenchem uma pesquisa validada de 15 itens para avaliar seus conhecimentos de habilidades em relação aos cuidados necessários para o controle do diabetes. As respostas às perguntas individuais da pesquisa são então usadas para direcionar os pacientes aos conteúdos audiovisuais que mediaram a adequação do conhecimento destes pacientes em relação ao controle da doença. O programa também inclui conteúdos audiovisuais obrigatórios, os vídeos utilizados neste programa variam em duração de 3 a 6 minutos. A plataforma de entrega registra as respostas dos pacientes às perguntas da pesquisa e os vídeos que assistem. 
Silva, et al. (2018), utilizou um simulador de baixo custo para capacitação de pacientes com Diabetes mellitus e/ou de seus cuidadores na aplicação de insulina. Para o desenvolvimento da pesquisa, foi realizado um levantamento de mercado com a finalidade de verificar quais simuladores as empresas do ramo recomendam para uso na capacitação de pacientes e/ou cuidadores para aplicação de insulina e seus preços de venda. Foram elencados como características esperadas nos simuladores: 1) possibilitar reconhecimento de todas as áreas recomendadas para aplicação de insulina; 2) treinamento da antissepsia da pele, da realização da prega subcutânea, da inserção da agulha no tecido subcutâneo com angulação de $90^{\circ}$ e $45^{\circ}$, da injeção no tecido subcutâneo e da manutenção da agulha no tecido subcutâneo por cinco segundos e 3) planejamento do rodízio dos pontos de aplicação. Para a validação desta tecnologia foram selecionados profissionais da área da saúde e educação, com pelo menos um certificado de especialização na área do estudo. A simulação clínica favorece a identificação de pontos críticos na execução das habilidades e no conhecimento e pode ser considerada uma ferramenta eficaz, relevante e inovadora para implementar, ensinar ou atualizar procedimentos (Daupin, et al., 2016).

Galdino, et al. (2019) visou construir e validar uma tecnologia educativa em formato de cartilha, para mediar o processo educativo de pessoas com diabetes. O processo de construção e validação da cartilha foi divido em dois momentos. Inicialmente, houve a construção do material educativo embasada nos itens do Questionário de Adesão ao Autocuidado com os Pés de Pacientes Diabéticos (QPED) e de bibliografias atualizadas sobre DM e pé diabético. Posteriormente, procedeu-se a validação do material educativo, com a validação de conteúdo, aparência e adequabilidade da cartilha, por meio da avaliação por juízes e, posteriormente, com a população-alvo. O conteúdo foi sumarizado em tópicos: O que é o pé diabético?; Como prevenir o pé diabético?; Como devo cuidar dos meus pés?; Onde devo procurar ajuda?. O público-alvo considerou que a tecnologia desenvolvida está adequada em relação à clareza e compreensão da linguagem e das ilustrações, sendo o conteúdo considerado relevante para transmissão de conhecimentos e sensibilização à promoção de mudança de hábitos.

A utilização de tecnologias educativas, para mediar o processo de aprendizagem do autocuidado em indivíduos com DM, contribuem para o controle dos níveis glicêmicos, da doença como um todo e na prevenção de complicações. Destarte, há necessidade de validação cientifica destes recursos, para que possam então, se caracterizar como estratégia efetiva para a adequação de conhecimento, assimilação e incorporação dos cuidados adequados para prevenção de complicações relacionadas a doença, bem como, e principalmente, para que estas tecnologias sejam adequadas para a realidade cultural e social do públicoalvo em questão (Galdino, et al., 2019).

Compreende-se a palavra "tecnologia" como o modo de fazer, o método e não, apenas, a tecnologia física em si. A utilização de tecnologias vem crescendo, gradativamente, como recurso nas práticas de cuidado e ensino de saúde (Áfio, et al., 2014). Neste contexto, esta revisão também buscou analisar a utilização destes recursos tecnológicos para mediar o processo educativo de pessoas com diabetes.

Os resultados da busca apontaram para a utilização de recursos tecnológicos mais simples como materiais impressos, em formato de cartilha, a qual apresenta-se como uma gerontotecnologia capaz de facilitar a compreensão do idoso sobre diferentes temáticas de saúde, por outro lado, pode ser ferramenta inviável para idosos de baixa escolaridade, pela incompreensão do conteúdo do material, sendo um ponto negativo da utilização de tecnologias impressas (Camacho, et al., 2014).

Outros tipos de tecnologia como as tecnologias de informação em saúde, as quais podem ser definidas pela utilização de aparelhos como smartphones, tablets, dentre outros (World Health Organization, 2011). Estes recursos têm se popularizado nos últimos anos e tornaram-se ferramentas de qualidade para a ampliação do acesso à informação, além de aproximar os profissionais ao idoso, proporcionando uma maior rede de apoio, por meio do diálogo, troca de experiências e dúvidas no tocante aos seus cuidados. Os dispositivos móveis com telas sensíveis ao toque, em aplicativos direcionados à saúde, entretenimento, transporte e mídias sociais contribuem para o bem-estar psicológico em idosos. No entanto, esses indivíduos podem apresentar 
dificuldades para aprender a usar estes recursos tecnológicos, necessitando de assistência técnica de familiares e amigos o que pode favorecer, efetivamente, a melhoria de seu aprendizado sobre o manuseio desses aparelhos (Chiu, et al.,2016).

\section{Considerações Finais}

Atividades educativas em enfermagem são relevantes para mediar o processo de saúde de um indivíduo, no que diz respeito a doenças crônicas, em especial ao diabetes. É de suma importância que os enfermeiros utilizem os recursos educativos e tecnológicos como grupos de apoio, cartilhas, aplicativos e ferramentas de simulação para promoção de ações e autocuidado, com o intuito de viabilizar o controle da doença, os hábitos saudáveis e a melhoria na qualidade de vida destes indivíduos.

A revisão das produções acerca da temática evidenciou que os estudos desenvolvidos por enfermeiros sobre a educação do paciente diabético como tema de pesquisa, ainda é pouco explorada em níveis de maior evidência científica, necessitando de aprofundamento nos métodos de pesquisa para as produções desenvolvidas com esta temática. Um dos pontos positivos encontrados nesta revisão, é o fato de que as produções nacionais foram predominantes neste estudo, o que pode ser justificado pelo cuidado dessas pessoas ser instituído pelas políticas públicas de atenção à saúde do idoso e pela instituição do programa HiperDia no âmbito da Atenção Primária do Sistema Único de Saúde (SUS) brasileiro.

Como contribuições para a pesquisa em enfermagem e em saúde pública espera-se que esta revisão integrativa, venha embasar futuros estudos que abordem a proposição de atividades educativas voltadas para o cuidado de pessoas com Diabetes mellitus, e principalmente para fundamentação da prática educativa e clínica de enfermeiros em prol da realização de ações de promoção da saúde, alicerçando a instituição de políticas públicas para a prevenção e/ou minimização das complicações advindas do Diabetes mellitus.

\section{Referências}

American Diabetes Association. (2021). Standards of medical care in Diabetes-2021. Diabetes Care. 44(1), S15-S33. https://doi.org/10.2337/dc21-S002

Áfio, A. C. E.; Balbino, A. C.; Alves, M. D. S.; Carvalho, L. V. de; Santos, M. C. L. \& Oliveira, N. R. (2014). Análise do conceito de tecnologia educacional em enfermagem aplicada ao paciente. Revista da Rede de Enfermagem do Nordeste.15(1), 158-16. http://www.periodicos.ufc.br/rene/article/view/3108

Baptista, M. H. B.; Dourado, F. C.; Gomides, D. dos S.; Teixeira, C. R. de S.; Freitas, M. C. F. de \& Pace, A. E. (2019). Education in Diabetes Mellitus for blood glucose self-monitoring: a quasi-experimental study. Rev Bras Enferm.72(6),1601-16088. http://dx.doi.org/10.1590/0034-7167-2018-0731

Brasil. Ministério da Saúde. Secretaria de Atenção à Saúde. Departamento de Atenção Básica. (2013). Estratégias para o cuidado da pessoa com doença crônica: diabetes mellitus. Brasília-DF. https://bvsms.saude.gov.br/bvs/publicacoes/estrategias_cuidado_pessoa_diabetes_mellitus_cab36.pdf

Camacho, A. C. L. F.; Abreu, L. T. de A.; Leite, B. S.; Mata, A. C. de O.; Louredo, D. dos S. \& Silva, R. P. (2014). Validação de cartilha informativa sobre idoso demenciado pelos enfermeiros e acadêmicos de enfermagem: estudo observacional-transversal. Revista de Pesquisa Cuidado é Fundamental Online. 6(1), 8-16. https://www.redalyc.org/articulo.oa?id=505750621003

Carvalho, S. L. de; Ferreira, M. A.; Medeiros, J. M. P.; Queiroga, A. C. F.; Moreira, T. R. \& Negreiros, F. D. da S. (2018). Conversation map: an educational strategy in the care of elderly people with diabetes mellitus. Rev Bras Enferm.71(2), 925-929. http://dx.doi.org/10.1590/0034-7167-2017-0064

Chiu, C. J.; Hu, Y. H.; Lin, D. C.; Chang, F. Y.; Chang, C. S. \& Lai, C. F. (2016). The attitudes, impact, and learning needs of older adults using apps on touchscreen mobile devices: Results from a pilot study. Computers in Human Behavior. 63,189-97. https://doi.org/10.1016/j.chb.2016.05.020

Daupin, J.; Atkinson, S.; Bédard, P.; Pelchat, V.; Lebel, D. \& Bussières, J. (2016). Medication errors room: a simulation to assess the medical, nursing and pharmacy staffs' ability to identify errors related to the medication-use system. J Eval Clin Pract. 22(6), 907-916. https://doi.org/10.1111/jep.12558

De La Fuente Coria, M. C.; Cruz-Cobo, C. \& Santi-Cano, M. J. (2020). Effectiveness of a primary care nurse delivered educational intervention for patients with type 2 diabetes mellitus in promoting metabolic control and compliance with long-term therapeutic targets: Randomised controlled trial. International Journal of Nursing Studies. 101, 103417. https://doi.org/10.1016/j.ijnurstu.2019.103417

Frota, S. S.; Lopes, L. V.; Onofre, M. R.; Dodou, H. D. \& Guedes, M. V. C. (2020). Aplicabilidade do modelo de adaptação de Roy no cuidado ao paciente diabético. Brazilian journal of health review. 3(4), 10699-10709. https://www.brazilianjournals.com/index.php/BJHR/article/view/15712

Galdino, Y. L. S.; Moreira, T. M. M.; Marques, A. D. B. \& Silva, F. A. A. da (2019). Validação de cartilha sobre autocuidado com pés de pessoas com Diabetes Mellitus. Revista Brasileira de Enfermagem. 72(3), 780-787. https://doi.org/10.1590/0034-7167-2017-0900

Hopia, H.; Latvala, E. \& Liimatainen, L. (2016). Reviewing the methodology of an integrative review. Scand J Caring Sci. 30 , 662- 669. https://doi.org/10.1111/scs.12327 
Kho, S. E. S.; Lim, S. G.; Hoi, W. H.; Ng, P. L.; Tan, L. \& Kowitlawakul, Y. (2019).The Development of a Diabetes Application for Patients With Poorly Controlled Type 2 Diabetes Mellitus. Comput Inform Nurs. 37(2), 99-106. https://doi.org/10.1097/cin.0000000000000485

Marques, M. B.; Coutinho, J. F. V.; Martins. M. C.; Lopes, M. V. de O.; Maia, J. C. \& Silva, M. J. da (2019). Intervenção edu cativa para a promoção do autocuidado de idosos com diabetes mellitus. Revista da Escola de Enfermagem da USP. 53, e03517. https://doi.org/10.1590/S1980-220X2018026703517

Melnyk, B. M.; Fineout-Overholt, E.; Stillwell, S. B. \& Williamson, K. M. (2010). Evidence-based practice: step by step: the seven steps of evidence-based practice. AJN The American Journal of Nursing. 110(1), 51-53. https://doi.org/10.1097/01.naj.0000366056.06605.d2

Mendes, K. D. S.; Silveira, R. C. de C. P. \& Galvão, C. M. (2008). Revisão integrativa: método de pesquisa para a incorporação de evidências na saúde e na enfermagem. Texto Contexto Enferm. 17(4), 758-764. https://doi.org/10.1590/S0104-07072008000400018

Powers, M. A.; Bardsley, J.; Cypress, M.; Duker, P.; Funnell, M. M.; Fischl, A. H.; Maryniuk, M. D.; Siminerio, L. \& Vivian, E. (2015). Diabetes selfmanagement education and support in type 2 diabetes: a joint position statement of the American Diabetes Association, the American Association of Diabetes Educators, and the academy of nutrition and dietetics. J. Acad. Nutr. Diet. 115(8), 1323-1234. https://doi.org/10.1016/j.jand.2015.05.012

Silva, J. P. da; Junior, G. A. P.; Meska, M. H. G. \& Mazzo, A. (2018). Construction and validation of a low-cost simulator for training patients with diabetes mellitus and/or their caregivers in insulin administration. Escola Anna Nery. 22(3), e20170387. https://doi.org/10.1590/2177-9465-EAN-2017-0387

Silva, W. I. dos S.; Oliveira, V. L. G. de; Leite, M. R.; Costa, R. E. A. R. da; Carvalho, D. de N. R. de; Aguiar, V. F. F. de; Costa, I. M. M.; Dergan, M. R. A.; Branco, I. B. C.; Amorim, M. G.; Camboim, L. F. R.; Fortes, M. M.; Rocha, C. E. M. C.; Medeiros, P. C. D. R.; Feitosa, N. L.; Nogueira, M. de A. \& Sá, A. M. M. (2020). Knowledge as a predictive factor for self-care adherence and glycemic control of people with diabetes. Research, Society and Development, 9(10), e1149108474. https://doi.org/10.33448/rsd-v9i10.8474

Sociedade Brasileira de Diabetes. (2019). Diretrizes da sociedade brasileira de diabetes (2019-2020). São Paulo: Clannad Editora científica. Diretrizes da Sociedade Brasileira de Diabetes 2019-2020.pdf (researchgate.net)

Smith, K. M.; Baker, K. M.; Bardsley, J. K.; McCartney, P. \& Magee, M. (2019). Redesigning Hospital Diabetes Education: A Qualitative Evaluation With Nursing Teams. J Nurs Care Qual. 34(2), 151-157. https://doi.org/10.1097/ncq.0000000000000349

Swanson, V. \& Maltinsky, W. (2019). Motivational and behaviour change approaches for improving diabetes management. Pract Diabet. 36(4), 121-125. https://doi.org/10.1002/pdi.2229

Torres, H. de C.; Cortez, D. N. \& Reis, I. A. (2016). Avaliação da Educação em Grupo de Diabetes na Atenção Primária à Saúde. Ciência y Enfermería. 22(3), 35-45. http://dx.doi.org/10.4067/S0717-95532016000300035.

Vest, B. M.; Hall, V. M.; Kahn, L. S.; Heider, A. R.; Maloney, N. \& Singh, R. (2017). Nurse perspectives on the implementation of routine telemonitoring for high-risk diabetes patients in a primary care setting. Primary Health Care Research \& Development. 18, 3-13. https://doi.org/10.1017/s1463423616000190

Wermeling, M.; Thiele-Manjali, U.; Koschack, J.; Lucius-Hoene, G. \& Himmel, W. (2014). Type 2 diabetes patients' perspectives on lifestyle counselling and weight management in general practice: a qualitative study. BMC Fam Pract.15, 97. https://doi.org/10.1186/1471-2296-15-97

Wilson, V. L. (2019). How to live well with diabetes: a comprehensive guide to taking control of your life with diabetes. London: Brown Book Group.

Wilson, V. L. (2021). Diabetes education to provide the necessary self-management skills. Br J Community Nurs. 26(4), 199-201. https://doi.org/10.12968/bjen.2021.26.4.199

Whittemore, R. \& Knafl, K. (2005). The integrative review: updated methodology. J Adv Nurs. 52(5), 546-553. https://doi.org/10.1111/j.13652648.2005.03621.x

World Health Organization. (2011). mHealth: new horizons for health through mobile technologies. Global Observatory for eHealth Series Vol 3. http://apps.who.int/iris/handle/10665/44607 